\title{
The Study on Building of Virtual Reality System in Large Surface Coal Mine
}

\author{
Baoying Ye, Nisha Bao, and Zhongke Bai \\ School of Land Science and Technology, China University of Geosciences, \\ Beijing, 100083, China \\ yebaoying@yahoo.cn
}

\begin{abstract}
The building of virtual reality system for the opencast mine is a complicated and systemic project. The building process of virtual system includes data collection, GIS geodabase, model designing and raster textures making. GIS data is from field survey, maps, and remote sensing images. The import phase is the building of kinds of models in different environment. This paper introduced several methods for the modeling different entity, such as dumps, pits, building in Industrial sites, and reclaimed vegetation. The dump models includes real and designed entity in GIS. The building models are built by GIS data and 3DMAX.The vegetation models is builtup in other special software. Based on the ArcGlobe, the kinds of models are integrated into the virtual reality system. ArcGlobe allows efficient display and query of raster data and GIS data, and it is integrated to function with the ArcGIS geodatabase while providing support for analysis in the geoprocessing environment.
\end{abstract}

\section{Introduction}

Virtual Reality Geographic Information System (VRGIS) is a technology, which combine Virtual reality with geographic information system (Batty M, 2000). It not only includes spatial analysis tools, but also can simulate landscape in the real world as well as in imaginary worlds with animation and audio function. A key part in VRGIS is considered as the acquire, display and processing of $3 \mathrm{D}$ data. There were a number of challenges in the study of environment virtualization through 3D space and time. (K. Morris, 2000). One of challenges is how to integrate different temporal and spatial resolutions data. Additionally, In the study of VRGIS, Modeling digital landscape is very complex phenomena, focus is only on the dynamic of terrain and human perception. (MILAP P., 2006).Moore et al. (1999) developed a 3D terrain visualization system by using Java and VRML.

The technology of aerial photography survey, laser scanning, field measurement and GPS improve the accuracy and speed in VRGIS. There is no doubt that it also provides great technical support for the study of mining VRGIS. Recently, there are many research on the study of VRGIS and 3D landscape visualization in mining environment. Shen Yanchun(2001) developed VRGIS system with MultiGen CreaterPro software based on the VEGA API program in large surface mine. $\mathrm{Wu}$ Yougen(2004) simulated land reclamation visualization in subsidence area of mining 
based on the VRML. 3D virtualization technology still be applied in land reclamation engineering(Jiang Quan, 2010 ).

The VRGIS technology for application mining land reclamation, referred to as landform models, industrial building models and rehabilitate vegetation models. Meanwhile, it is critical for the system on the data integration and 3D dynamic graphics. Therefore, this paper is aimed to analyze the process of mining 3D visualization, and describe VRGIS system developed to store, access and visualize diverse environmental data by 3D model based on ArcGlobe.

\section{System Development}

An important criterion for assessing the suitability of visualization systems for land reclamation is the capability of simulating different kinds of movement in the landscape, such as open-pit, dump, industrial sites, reclaimed vegetation, etc. Therefore, the modeling design which is composed of terrain, building and vegetation is one of most significant part for building system.

\subsection{Field Data Collection}

Field survey is to collect model information by taking pictures in dump, opencast, and industrial sites. All those data can provide the size, shape texture of object for building landscape model.

\subsection{Land Reclamation Investigation}

Because of large area and different types of land cover in surface coal mine, remote sensing is a useful tool for field collected observations, so the reclaimed vegetation data is extracted from high resolution image. In this study, the QUICKBIRD image

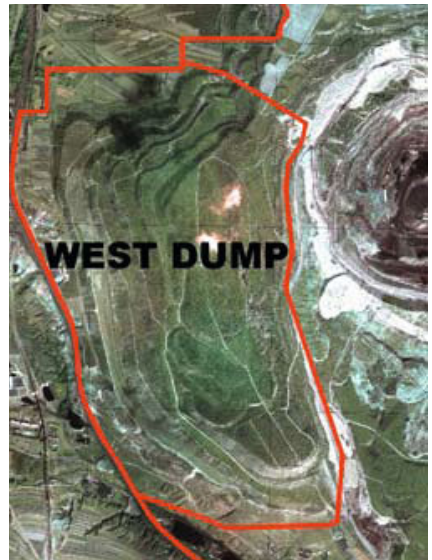

a. The quickbird image of dump

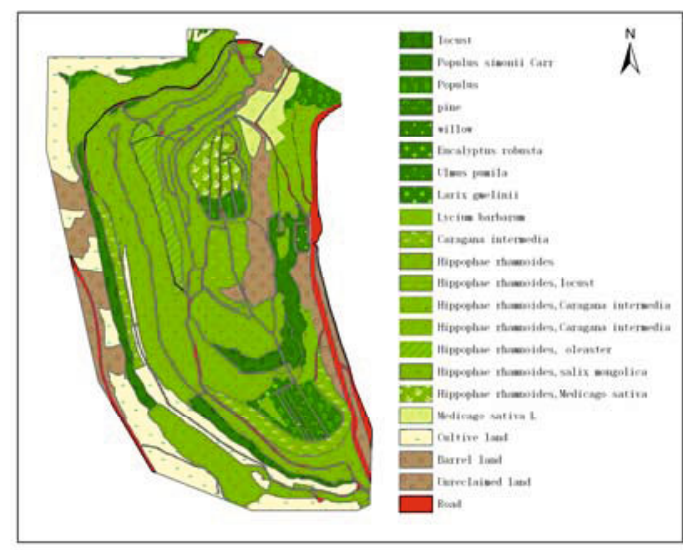

b. The landuse map of dump from quickbird image

Fig. 1. The QUICKBIRD image and landuse map 
(0.6m) of 2006 is used to investigate the reclamation information, including the vegetation type, time, location, and so on (Figure 1). All the data are prepared to compile into database.

\subsection{Model Design}

The visualization system research is mainly focus on the $3 \mathrm{~d}$ landscape modeling, which is considered as the challenge (1) building up box model effectively with data of GIS or CAD, and topographic data; (2) generating real time animations of complex scenes represented as $3 \mathrm{~d}$ planar polygons surface with remote sensing image or aero photograph, However , it is posed by the high prices and time-consuming, comparing other method; (3) establishing the geometric surface model with $3 \mathrm{~d}$ mesh point using laser scanning and measurement of ground movement[4].

Recently, the commercial 3D software, such as 3D MAX, Maya, Blender, SketchUp released by Google, are widely used in landscape modeling based on map and photograph. Some software, such as tree factory, Onyx Tree, Amap are mainly used in vegetation modeling. In this study, according to complex scene and various types of landscape, many modeling way are applied to meet the practical need.

\subsubsection{Modelling of Dump}

Dump refers to a place for disposal of waste in surface mining process. At the beginning of the exploration, the dump is designed, including location, area, step, slope and platform. Therefore, during the modeling of dump, one part is to build from previous design data which is ideal dump model, the other part is to build from practice topography data.

The terrain data is usually captured by aerial survey or field measurement, which is the format of vector contour, and the TIN and GRID are also the models for presenting real terrain.

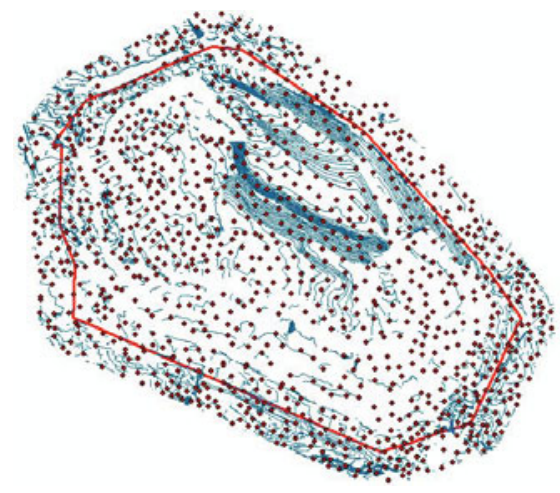

a. The $2 \mathrm{D}$ vector model of dump

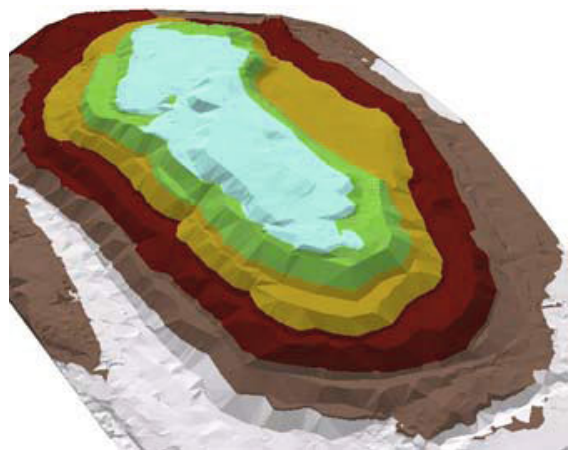

b. The 3D TIN model of dump

Fig. 2. The $2 \mathrm{D}$ and $3 \mathrm{D}$ model of dump 
The original terrain data provided by mining company are used to be the AUTOCAD format, and without projection. So firstly, the data in CAD should be converted to that in GIS, and then transform it to the proper projection. In this study, the software of FME is used to complete the data conversion. The data of CAD format is converted into that of shape file format, and define the projection in the ArcGIS, After this previous data processing, terrain data in GIS is to build TIN model(Figure 2).

According to the design of dump, we could get the data of the dump boundary, width, height, and slope, and then generate contour lines of dump automatically. Finally, the Tin model is generated from the contour lines. The dump volume is easily to be calculated from this 3D model (Figure 3).

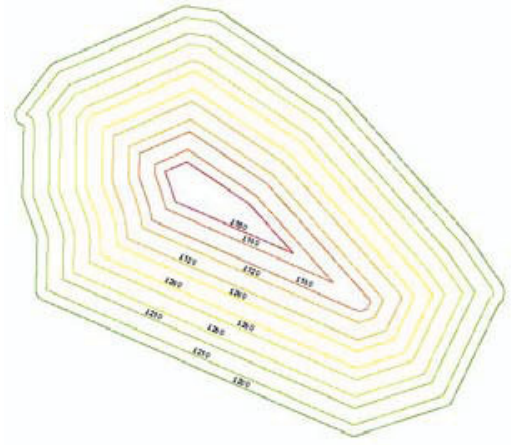

a. The designed $2 \mathrm{D}$ vector model of dump

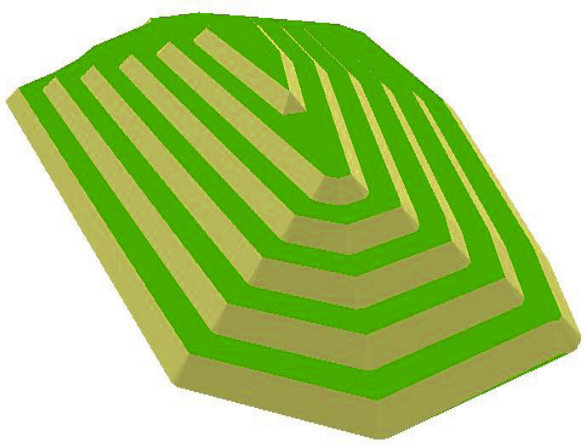

b. The designed 3D TIN model of dump

Fig. 3. The designed 2D and 3D model of dump

\subsubsection{Modeling of Pit}

During the waste dumping process, mine waste in-pit disposal will be transited from base to the peak of the pit; meanwhile, the excavated face will be pushed forward after the forming of the mining bench.

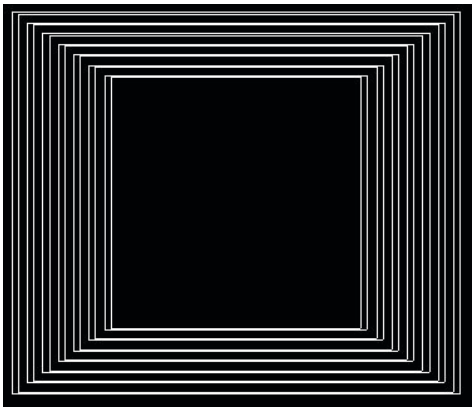

a. The contours model of inner dump and stope

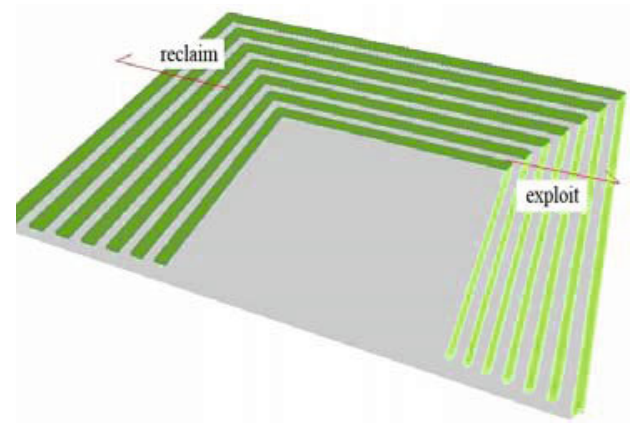

b. The 3D TIN model of inner dump and stope

Fig. 4. The models of inner dump and stope 
The slope and width of waste dump is completely different from that of open-pit, The contours of stope-boundary, pit and waste dumping could be designed by ArcINFO AML program, and then to be overplayed to modify the related parameters. The tin model (Figure 4) was generated from contours.

\subsubsection{Modeling of Industrial Sites}

There are various buildings in industrial sites, it will cost much time when the model is built by 3DMAX or Maya software. The system of land reclamation and ecological restoration is interested in the visualization in pit, waste dump and reclaimed vegetation. Therefore, in this study, for some landmark buildings, we adopted conventional manual modeling method to build model. However, for most buildings, we used the $3 \mathrm{~d}$ model by GIS software.

\subsubsection{Modeling of Reclaimed Vegetation}

In the vegetation modeling, the field survey photo is used to model the vegetation based on the Onyx Tree. Amap download from 3DMAX. Additionally, the texture from remote sensing image is also can be used to render plants and vegetation, and then to be located in each waste dump according to the reclamation.

\subsection{System Integration}

In virtual reality system technology, systems integration is the process of linking together different model physically or functionally based on uniform platform. System integration is also about development function. Due to the complex of the 3D engine, in this study, ArcGlobe software released by ESRI is used to integrate system, which is helpful for the virtual terrain model and accurate geographic location. Most types of raster and vector data that are spatially referenced can be loaded into a globe. ArcGlobe can display substantial amounts of data quickly and effectively. The data can be widespread or limited in extent with highly detailed or coarse resolution. It also has the function of overlay, viewshed and buffer analysis, and adding outside model through 3D label. We can get more details from Figure 5.

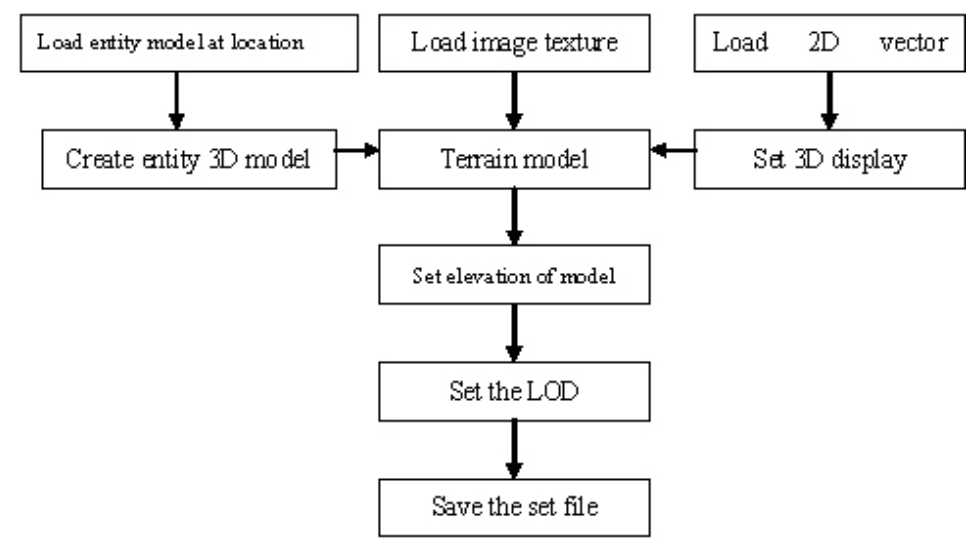

Fig. 5. The process diagram of integrating models into the VR system 


\section{Conclusion}

This study illustrates the issues of modeling in land reclamation and ecological restoration, as well as the optimal allocation of land use resource. In conclusion: (1) For the study of large landscape modeling, The problem which should be handled is the resource and processing of high resolution data. It is necessary to data conversion from AUTOCAD format to GIS format for making terrain model; (2) There are both raw and artificial landscape in study area. For better 3D visual perception, the high resolution remote sensing image are widely applied. However, it is difficult for most software to convert image to JPEG or TIFF format, and match the local landscape and landform. Because of mass of data, map division should be used during the data process to bypass the weakness. (3) The 3D modeling is the most significant part in the virtual reality system; (4) Model making by other software should be positioned according to given coordinate. Therefore, the virtual reality system should be building based on GIS.

\section{References}

[1] Batty M., et al.: Visualizing the City : Communicating Urban Design to Planners and Decision - Makers (2000), http: / / www. Casa.ucl.ac.uk/visualcities.pdf

[2] Wu, Y., Chen, Q., Liu, C.: 3D landscape visualization in land reclamation area based on GIS. Ming Technology 4(4), 48-50 (2004)

[3] Shen, Y.-c., Zhang, Y.-d., Cai, Q.-X.: Open-pit mine ecologocal reconstruction VR simulation system. Industrial Minerals and Porocessing 11, 8-10 (2001)

[4] Tao, V.: Data collection and 3D object reconstruction, Large -scale 3D data integration Problems and challenges). In: Bentley International User Conference (2004)

[5] Li, J., Bai, Z.: Land reclamation and ecological restoration -a case of Pingshuo large suface coal mine, pp. 28-33. Science Press, Beijing (2001)

[6] Jiang, Q., Chen, J., Yang, H.: Applica tion of Three - Dimension Visual Technology in Reclamation of Pingguo Bauxite Mine. Mining research and development 30(1), 77-79 (2010)

[7] Moore, K., Dykes, J., Wood, J.: Using JAVA to interact with geo-referenced VRML within a virtual field course. Computers and Graphics 25, 1125-1136 (1999)

[8] Punia, M., Pandey, D.: 3D Landscape Modelling Using Java 3D/Vrml. Journal of the Indian Society of Remote Sensing 34(4) (2006)

[9] Morris, K., Hillb, D., Moore, A.: Mapping the environment through three-dimensional space and time. Computers, Environment and Urban Systems 24, 435-450 (2000) 